\title{
Effect of Variable permeability, concentration and viscous dissipation with Chemical reaction and Oscillating temperature on Unsteady MHD Walter's-B viscoelastic dusty fluid past over inclined porous plate with heat radiation
}

\author{
Nidhi Pandya ${ }^{1}$, Mohammad Suleman Quraishi ${ }^{2 *}$ \\ ${ }^{1,2}$ Department of Mathematics and Astronomy, University of Lucknow, Lucknow, U.P., India \\ "Corresponding Author: sulemanformaths86@gmail.com,Tel.: +91-9389204170
}

Available online at: www.isroset.org

Accepted 18/Aug/2018, Online 30/Aug/2018

\begin{abstract}
In this paper we have investigated the effects of radiation, chemical reaction, viscous dissipation and variable permeability for an unsteady MHD (Magnetohydrodynamic) Walter's-B viscoelastic dusty flow of an incompressible viscous and electrically conducting dusty fluid past a continuously moving inclined porous plate with mass transfer and oscillating temperature. The Governing partial differential equations of fluid profiles are solved numerically by Crank-Nicolson method and results are discussed with graphs.
\end{abstract}

Keywords: MHD, Chemical reaction, thermal radiation, porous medium, Heat and mass transfer, Crank-Nicolson Method.

\section{INTRODUCTION}

Radiation and Chemical reaction effect on MHD flows arise in many areas of engineering and applied physics. The study of such flow has application in MHD generators, chemical-engineering, nuclear reactors, geothermal energy, reservoir engineering and astrophysical studies. In nature, the assumption of the pure fluid is rather impossible. The presence of foreign mass in the fluid plays an important role in flow of fluid. In last many decades, large number of investigation of unsteady MHD flow of NonNewtonian fluid have been taken place because study of heat and mass transfer in Non-Newtonian fluid has application in different field of engineering and science like milk processing, chemical and process engineering, blood oxygenators, mixing mechanisms and dissolution process etc. There exist many viscoelastic fluids and their models to study Non-Newtonian fluids like second grade viscoelastic fluids, third grade viscoelastic fluids, micro polar fluids, Rivlin Ericksen model, Maxwell model and Walter's-B model etc.The Walter's-B model or viscoelastic fluids had been developed to simulate accurately tribiological, biotechnological and many complex polymeric viscous fluid possessing short time elastic effects. Steady flow and heat transfer in a viscoelastic fluid between two coaxial rotating disks was analyzed by Bastia and Rath [1]. It is investigated Heat transfer from flow of an elastico-viscous fluid by Raptis and Takhar [2]. Hall Effect on MHD mixed convection flow of a viscoelastic fluid past an infinite vertical porous plate with mass transfer and radiation is studied by Chaudhary and Jain [3]. Non similar solutions for heat and mass transfer flow in an electrically conducting viscoelastic fluid over a stretching sheet saturated in a porous medium with suction/blowing by Rajgopal, Veena, Pravin [4]. It is also analyzed that Momentum and heat transfer in the magnetohydrodynamic stagnation point flow of a viscoelastic fluid towards a stretching surface by Dholey, Gupta and Mahapatra [5]. Further it has examined Finite difference analysis of transient free convection with mass transfer on an isothermal vertical flat plate by Ganesan and Soundalgekar [6]. SoretDufour and radiation effects on unsteady MHD flow past an impulsively started inclined porous plate with variable temperature and mass diffusion by Pandya and Shukla [7]. Soret-Dufour and radiation effects on 
unsteady MHD flow over an inclined porous plate embedded in porous medium with viscous dissipation by Pandya and Shukla [8]. Radiation effects on unsteady free convection heat and mass transfer in a Walter's-B viscoelastic flow past an impulsively started vertical plate by Rao, Prasad, Vishwanath and Vasu [9]. Numerical Simulation of Soret-Dufour and Radiation effects on Unsteady MHD flow of Viscoelastic Dusty fluid over Inclined Porous Plate by Pandya and Yadav [10].

\section{Mathematical ANalysis}

An unsteady flow of a viscoelastic Walter's-B incompressible electrically conducting fluid past an impulsively started infinite vertical porous plate with variable permeability, oscillating temperature and variable mass diffusion with radiation, chemical reaction and viscous dissipation are analyzed. Plate is embedded in porous medium, $\mathrm{x}^{\prime}-$ axis and $\mathrm{y}^{\prime}-$ axis are taken along and normal to plate respectively. Initially the plate and fluid are at the same temperature and concentration $\mathrm{T}_{\infty}{ }^{\prime}$ and $\mathrm{C}_{\infty}{ }^{\prime}$ respectively. At the time $\mathrm{t}^{\prime}>0$ plate is given in motion along $\mathrm{x}^{\prime}$ direction with constant velocity $u_{0}$. A transverse magnetic field $B_{0}$ is considered normal to the direction of flow. Magnetic Reynold number and transversely applied magnetic field are very small therefore induced magnetic field is negligible [11].

Due to infinite length in $x$ '-direction the flow variables are functions of y' and t' only. Under the above

Assumptions, the governing boundary layer equations with Boussinesq's approximation are

Continuity Equation:

$$
\frac{\partial v^{\prime}}{\partial y^{\prime}}=0 \Rightarrow v^{\prime}=-v_{0}(\text { Say }, \text { Constant }=1)
$$

Momentum Equations:
Hence the objective of this paper is to analyze the effects of variable permeability, radiation and chemical reaction on unsteady Walter's-B viscoelastic MHD dusty flow past a semi infinite inclined porous plate embedded in porous media with oscillating temperature and viscous dissipation. Non-dimensional form of partial differential equations of flow has been solved by applying Crank-Nicolson implicit finite difference method. Velocity, temperature and concentration profiles are discussed through graphs for different values of parameters.

$$
\begin{aligned}
& \frac{\partial \mathrm{u}^{\prime}}{\partial \mathrm{t}^{\prime}}+\mathrm{v}^{\prime} \frac{\partial \mathrm{u}^{\prime}}{\partial \mathrm{y}^{\prime}}=\mathrm{v} \frac{\partial^{2} \mathrm{u}^{\prime}}{\partial \mathrm{y}^{\prime 2}}+\mathrm{g} \beta \operatorname{Cos} \lambda\left(\mathrm{T}^{\prime}-\mathrm{T}_{\infty}^{\prime}\right) \\
& -\frac{\sigma \mathrm{B}_{0}{ }^{2} \mathrm{u}^{\prime}}{\rho}-\frac{\mathrm{v \textrm {u } ^ { \prime }}}{\mathrm{K}^{\prime}}+\mathrm{g} \beta^{*} \operatorname{Cos} \lambda\left(\mathrm{C}^{\prime}-\mathrm{C}_{\infty}^{\prime}\right) \\
& +\frac{\mathrm{K}_{1} \mathrm{~N}_{0}\left(\mathrm{~V}-\mathrm{u}^{\prime}\right)}{\rho}-\mathrm{k}_{0} \frac{\partial^{3} \mathrm{u}^{\prime}}{\partial \mathrm{y}^{\prime 2} \partial \mathrm{t}^{\prime}} \\
& \mathrm{m}_{1} \frac{\partial \mathrm{V}}{\partial \mathrm{t}^{\prime}}=\mathrm{K}_{1}\left(\mathrm{u}^{\prime}-\mathrm{V}\right)
\end{aligned}
$$

Energy equation:

$\rho \mathrm{C}_{\mathrm{p}}\left(\frac{\partial \mathrm{T}^{\prime}}{\partial \mathrm{t}^{\prime}}+\mathrm{v}^{\prime} \frac{\partial \mathrm{T}^{\prime}}{\partial \mathrm{y}^{\prime}}\right)=\mathrm{k} \frac{\partial^{2} \mathrm{~T}^{\prime}}{\partial \mathrm{y}^{\prime 2}}+\mu\left(\frac{\partial \mathrm{u}^{\prime}}{\partial \mathrm{y}^{\prime}}\right)^{2}-\frac{\partial \mathrm{q}_{\mathrm{r}}}{\partial \mathrm{y}^{\prime}}$

Equation of Continuity for mass transfer:

$$
\frac{\partial \mathrm{C}^{\prime}}{\partial \mathrm{t}^{\prime}}+\mathrm{v}^{\prime} \frac{\partial \mathrm{C}^{\prime}}{\partial \mathrm{y}^{\prime}}=\mathrm{D} \frac{\partial^{2} \mathrm{C}^{\prime}}{\partial \mathrm{y}^{\prime 2}}-\mathrm{k}_{\mathrm{r}}\left(\mathrm{C}^{\prime}-\mathrm{C}_{\infty}^{\prime}\right)
$$

Where $g$ is gravitational acceleration, $\beta$ is the volumetric coefficient of thermal expansion, $\beta^{*}$ is the coefficient of volume expansion for mass transfer, $\mathrm{K}$ ' is the permeability of porous medium, $\sigma$ is the electrical conductivity of the fluid, $T^{\prime}$ is the dimensional temperature, $v$ is the kinematic viscosity, is viscosity, $\rho$ is the fluid density, $B_{0}$ is magnetic induction, $q_{\mathrm{r}}$ is radiative heat flux in y'-direction, $D$ is mass diffusion coefficient, $\mathrm{k}_{\mathrm{r}}$ is chemical reaction rate constant, $k_{0}$ 
Walter's-B visco-elastic parameter, $\mathrm{C}_{\mathrm{p}}$ is specific heat at constant pressure, $k$ is the thermal conductivity of the fluid, C' and T'are dimensional concentration and temperature of fluid and plate, $V$ is the velocity of dust particles, $C_{\infty}{ }^{\prime}$ and $\mathrm{T}_{\infty}{ }^{\prime}$ are concentration and temperature of free stream respectively, $q_{r}$ is radiative heat flux along $y^{\prime}$-axis, $N_{0}$ is the number density of the dust particles which is taken to be constant, $K_{1}$ is the stokes resistance coefficient, $m_{1}$ is the mass of dust particle, $k_{r}$ is chemical reaction parameter.

Initial and boundary conditions are as follows:

$$
\left.\begin{array}{c}
\mathrm{t}^{\prime} \leq 0 ; \quad \mathrm{u}^{\prime}=0, \quad \mathrm{~V}=0, \quad \mathrm{~T}^{\prime}=0, \quad \mathrm{C}^{\prime}=0, \mathrm{v}_{0}=1 \quad \forall \mathrm{y}^{\prime} \\
\mathrm{t}^{\prime}>0 ; \quad \mathrm{u}^{\prime}=\mathrm{u}_{0}, \mathrm{~V}=\mathrm{u}_{0}, \mathrm{~T}^{\prime}=\mathrm{T}_{\infty}^{\prime}+\left(\mathrm{T}_{\mathrm{w}}^{\prime}-\mathrm{T}_{\infty}^{\prime}\right) \operatorname{Cos} \omega^{\prime} \mathrm{t}^{\prime}, \\
\mathrm{C}^{\prime}=\mathrm{C}_{\infty}^{\prime}+\left(\mathrm{C}_{\mathrm{w}}^{\prime}-\mathrm{C}_{\infty}^{\prime}\right) / \mathrm{At}^{\prime} \quad \text { at } \mathrm{y}^{\prime}=0 \\
\mathrm{u}^{\prime} \rightarrow 0, \mathrm{~V} \rightarrow 0, \quad \mathrm{~T}^{\prime} \rightarrow \mathrm{T}_{\infty}^{\prime}, \quad \mathrm{C}^{\prime} \rightarrow \mathrm{C}_{\infty}^{\prime} \quad \text { as } \mathrm{y}^{\prime} \rightarrow \infty
\end{array}\right\}
$$

Where, $\mathrm{A}=\frac{\mathrm{u}_{0}{ }^{2}}{\mathrm{v}}, \quad \mathrm{T}_{\mathrm{w}}^{\prime}$ and $\mathrm{C}_{\mathrm{w}}^{\prime}$ temperature and concentration of plate respectively.

Radiative heat flux term using the Roseland approximation is given by

$$
\mathrm{q}_{\mathrm{r}}=-\frac{4 \sigma^{*}}{3 \mathrm{k}_{\mathrm{m}}} \frac{\partial \mathrm{T}^{\prime 4}}{\partial \mathrm{y}^{\prime}}
$$

Here Stefan Boltzmann constant and absorption coefficient are $\sigma^{*}$ and $\mathrm{k}_{\mathrm{m}}$ respectively. In this case temperature difference are very-very small within flow, such that $\mathrm{T}^{\prime 4}$ can be expressed linearly with temperature. It is realized by expanding in a Taylor series about $\mathrm{T}_{\infty}{ }^{\prime}$ and neglecting higher order terms, so

$\mathrm{T}^{\prime 4} \cong 4 \mathrm{~T}_{\infty}{ }^{3} \mathrm{~T}^{\prime} \square-\square 3 \mathrm{~T}_{\infty}{ }^{4}$

Using equation (7) and (8), equation (9) becomes

$$
\begin{aligned}
& \frac{\partial \mathrm{T}^{\prime}}{\partial \mathrm{t}^{\prime}}+\mathrm{v}^{\prime} \frac{\partial \mathrm{T}^{\prime}}{\partial \mathrm{y}^{\prime}}=\frac{\mathrm{k}}{\rho \mathrm{C}_{\mathrm{p}}} \frac{\partial^{2} \mathrm{~T}^{\prime}}{\partial \mathrm{y}^{\prime 2}} \\
& +\frac{16 \sigma \mathrm{T}_{\infty}^{\prime 3}}{3 \mathrm{k}_{\mathrm{m}}} \frac{\partial^{2} \mathrm{~T}^{\prime}}{\partial \mathrm{y}^{\prime 2}} \frac{1}{\rho \mathrm{C}_{\mathrm{p}}}+\mu\left(\frac{\partial \mathrm{u}^{\prime}}{\partial \mathrm{y}^{\prime}}\right)^{2}
\end{aligned}
$$

In order to obtain non dimensional form of governing equations, we introduce following quantities:

$$
\begin{aligned}
& \mathrm{u}=\frac{\mathrm{u}^{\prime}}{\mathrm{u}_{0}}, \mathrm{v}=\frac{\mathrm{V}}{\mathrm{u}_{0}}, \mathrm{t}=\frac{\mathrm{t}^{\prime} \mathrm{u}_{0}^{2}}{\mathrm{v}}, \mathrm{y}=\frac{\mathrm{y}^{\prime} \mathrm{u}_{0}}{\mathrm{v}}, \theta=\frac{\mathrm{T}^{\prime}-\mathrm{T}_{\infty}^{\prime}}{\mathrm{T}_{\mathrm{w}}^{\prime}-\mathrm{T}_{\infty}^{\prime}}, \\
& \mathrm{C}=\frac{\mathrm{C}^{\prime}-\mathrm{C}_{\infty}^{\prime}}{\mathrm{C}_{\mathrm{w}}^{\prime}-\mathrm{C}_{\infty}^{\prime}}, \quad \mathrm{G}_{\mathrm{m}}=\frac{v \mathrm{~g} \beta^{*}\left(\mathrm{C}_{\mathrm{w}}^{\prime}-\mathrm{C}_{\infty}^{\prime}\right)}{\mathrm{u}_{0}{ }^{3}}, \\
& \mathrm{G}_{\mathrm{r}}=\frac{v \mathrm{~g} \beta\left(\mathrm{T}_{\mathrm{w}}^{\prime}-\mathrm{T}_{\infty}^{\prime}\right)}{\mathrm{u}_{0}{ }^{3}}, \mathrm{~K}=\frac{\mathrm{u}_{0}{ }^{2}}{v^{2}} \mathrm{~K}^{\prime}, \mathrm{S}_{\mathrm{c}}=\frac{v}{\mathrm{D}} \text {, } \\
& \mu=\rho v, \mathrm{P}_{\mathrm{r}}=\frac{\mu \mathrm{C}_{\mathrm{p}}}{\mathrm{k}}, \mathrm{M}=\frac{\sigma \mathrm{B}_{0}{ }^{2} v}{\rho \mathrm{u}_{0}{ }^{2}}, \mathrm{R}=\frac{4 \sigma \mathrm{T}_{\infty}^{\prime}}{\mathrm{k}_{\mathrm{m}} \mathrm{k}}, \\
& \mathrm{Ec}=\frac{\mathrm{u}_{0}{ }^{2}}{\left(\mathrm{~T}_{\mathrm{w}}^{\prime}-\mathrm{T}_{\infty}^{\prime}\right) \mathrm{C}_{\mathrm{p}}}, \mathrm{K}_{\mathrm{r}}=\frac{\mathrm{k}_{\mathrm{r}} \nu}{\mathrm{u}_{0}{ }^{2}}, \mathrm{~B}_{1}=\frac{\nu \mathrm{K}_{1} \mathrm{~N}_{0}}{\rho \mathrm{u}_{0}{ }^{2}} \text {, } \\
& \mathrm{B}=\frac{\mathrm{m}_{1} \mathrm{u}_{0}{ }^{2}}{\mathrm{~V} \mathrm{~K}_{1}}, \mathrm{~A}=\frac{\mathrm{u}_{0}{ }^{2}}{\mathrm{v}}, \omega=\frac{\omega^{\prime} v}{\mathrm{u}_{0}{ }^{2}}, \Gamma=\frac{\mathrm{k}_{0} \mathrm{u}_{0}{ }^{2}}{v^{2}}
\end{aligned}
$$

$\mathrm{G}_{\mathrm{r}}, \mathrm{G}_{\mathrm{m}}, \mathrm{S}_{\mathrm{c}}, \mathrm{P}_{\mathrm{r}}, \mathrm{M}, \mathrm{Ec}, \mathrm{R}, \sigma$ and $\Gamma$ are Grashoff number, Solutal Grashoff number, Schmidt number, Prandtl number, Magnetic parameter, Eckert Number, Radiation parameter, electrical conductivity and viscoelastic parameter respectively.

Using above quantities of equation (10), we get non dimensional form of equations (2), (3), (9) and (5) respectively.

$$
\begin{aligned}
& \frac{\partial \mathrm{u}}{\partial \mathrm{t}}-\frac{\partial \mathrm{u}}{\partial \mathrm{y}}=\frac{\partial^{2} \mathrm{u}}{\partial \mathrm{y}^{2}}+\mathrm{G}_{\mathrm{r}} \operatorname{Cos} \lambda \theta+\mathrm{G}_{\mathrm{m}} \operatorname{Cos} \lambda \mathrm{C} \\
& +\mathrm{B}_{1}(\mathrm{v}-\mathrm{u})-\Gamma \frac{\partial^{3} \mathrm{u}}{\partial \mathrm{y}^{2} \partial \mathrm{t}}-\left(\mathrm{M}+\frac{1}{\mathrm{~K}}\right) \mathrm{u}
\end{aligned}
$$

$$
\mathrm{B} \frac{\partial \mathrm{v}}{\partial \mathrm{t}}=\mathrm{u}-\mathrm{v}
$$

$$
\begin{aligned}
& \frac{\partial \theta}{\partial \mathrm{t}}-\frac{\partial \theta}{\partial \mathrm{y}}=\frac{1}{\mathrm{P}_{\mathrm{r}}}\left(1+\frac{4 \mathrm{R}}{3}\right) \frac{\partial^{2} \theta}{\partial \mathrm{y}^{2}}+\mathrm{Ec}\left(\frac{\partial \mathrm{u}}{\partial \mathrm{y}}\right)^{2} \\
& \frac{\partial \mathrm{C}}{\partial \mathrm{t}}-\frac{\partial \mathrm{C}}{\partial \mathrm{y}}=\frac{1}{\mathrm{~S}_{\mathrm{c}}} \frac{\partial^{2} \mathrm{C}}{\partial \mathrm{y}^{2}}-\mathrm{K}_{\mathrm{r}} \mathrm{C}
\end{aligned}
$$

Where the permeability of porous medium $\mathrm{K}$ varies with $\mathrm{y}$ that is, $\mathrm{K}$ is inversely proportional to $\mathrm{y}$ such that 
it lie between 0 and 1 and it decreases with increase in y.

And dimensionless boundary conditions are

$\left.\begin{array}{l}\mathrm{t} \leq 0 ; \quad \mathrm{u}=0, \quad v=0, \quad \quad \mathrm{~T}=0, \quad \mathrm{C}=0 \\ \mathrm{t}>0 ; \quad \mathrm{u}=1, \mathrm{v}=1, \quad \theta=\operatorname{Cos} \omega t, \quad \mathrm{C}=1 / \mathrm{t} \quad \text { at } \quad \mathrm{y}=0 \\ \mathrm{u} \rightarrow 0, \mathrm{v} \rightarrow 0, \mathrm{~T} \rightarrow 0, \quad \mathrm{C} \rightarrow 0 \quad \text { as } \quad \mathrm{y} \rightarrow \infty\end{array}\right\}$

\section{METHOD OF SOLUTION}

$\frac{u_{i, j+1}-u_{i, j}}{\Delta t}-\frac{u_{i+1, j}-u_{i, j}}{\Delta y}=$

$\left(\frac{u_{i-1, j}-2 u_{i, j}+u_{i+1, j}+u_{i-1, j+1}-2 u_{i, j+1}+u_{i+1, j+1}}{2(\Delta y)^{2}}\right)$

$+G_{r} \operatorname{Cos} \lambda\left(\frac{\theta_{i, j+1}+\theta_{i, j}}{2}\right)+G_{m} \operatorname{Cos} \lambda\left(\frac{C_{i, j+1}+C_{i, j}}{2}\right)$

$-M\left(\frac{u_{i, j+1}+u_{i, j}}{2}\right)-(1 / \Delta y)\left(\frac{u_{i, j+1}+u_{i, j}}{2}\right)$

$-\Gamma\left(\frac{u_{i-1, j}-2 u_{i, j}+u_{i+1, j}+u_{i-1, j+1}-2 u_{i, j+1}+u_{i+1, j+1}}{2(\Delta y)^{2} \Delta t}\right)$

$-B_{1}\left(\frac{v_{i, j+1}+v_{i, j}}{2}-\frac{u_{i, j+1}+u_{i, j}}{2}\right)$

$B \frac{v_{i, j+1}-v_{i, j}}{\Delta t}=\left(\frac{u_{i, j+1}+u_{i, j}}{2}-\frac{v_{i, j+1}+v_{i, j}}{2}\right)$ $\frac{\theta_{i, j+1}-\theta_{i, j}}{\Delta t}-\frac{\theta_{i+1, j}-\theta_{i, j}}{\Delta y}=-\left(\frac{u_{i, j+1}+u_{i, j}}{2}\right)$

$+\mathrm{Ec}\left(\frac{\mathrm{u}_{\mathrm{i}+1, \mathrm{j}^{-} \mathrm{u}_{\mathrm{i}, \mathrm{j}}}}{\Delta \mathrm{y}}\right)^{2}+\frac{1}{\mathrm{P}_{\mathrm{r}}}\left(1+\frac{4 \mathrm{R}}{3}\right)$

$\left(\frac{\theta_{i-1, j}-2 \theta_{i, j}+\theta_{i+1, j}+\theta_{i-1, j+1}-2 \theta_{i, j+1}+\theta_{i+1, j+1}}{2(\Delta y)^{2}}\right)$

$\frac{C_{i, j+1}-C_{i, j}}{\Delta t}-\frac{C_{i+1, j}-C_{i, j}}{\Delta y}=-K_{r}\left(\frac{C_{i, j+1}+C_{i, j}}{2}\right)$

$+\frac{1}{S_{c}}\left(\frac{C_{i-1, j}-2 C_{i, j}+C_{i+1, j}+C_{i-1, j+1}-2 C_{i, j+1}+C_{i+1, j+1}}{2(\Delta y)^{2}}\right)$

Corresponding boundary and initial conditions are

$$
\begin{aligned}
& \mathrm{u}_{\mathrm{i}, 0}=0, \quad \mathrm{v}_{\mathrm{i}, 0}=0, \\
& \theta_{\mathrm{i}, 0}=0, \quad \mathrm{C}_{\mathrm{i}, 0}=0 \quad \forall \mathrm{i} \\
& \mathrm{u}_{0, \mathrm{j}}=1, \quad \mathrm{v}_{0, \mathrm{j}}=1, \\
& \theta_{0, \mathrm{j}}=\operatorname{Cos} \omega^{*} \mathrm{j}^{*} \Delta \mathrm{t}, \mathrm{C}_{0, \mathrm{j}}=1 /(\mathrm{j} * \Delta \mathrm{t}) \\
& \mathrm{u}_{\mathrm{L}, \mathrm{j}} \rightarrow 0, \quad \mathrm{v}_{\mathrm{L}, \mathrm{j}} \rightarrow 0, \\
& \theta_{\mathrm{L}, \mathrm{j}} \rightarrow 0, \quad \mathrm{C}_{\mathrm{L}, \mathrm{j}} \rightarrow 0
\end{aligned}
$$

Here, index $\mathrm{i}$ refers to $\mathrm{y}$ and $\mathrm{j}$ to time, $\Delta \mathrm{t}=\mathrm{t}_{\mathrm{j}+1}-\mathrm{t}_{\mathrm{j}}$ and $\Delta \mathrm{y}=\mathrm{y}_{\mathrm{i}+1}-\mathrm{y}_{\mathrm{i}}$. For known values of $\mathrm{u}, \mathrm{w}$, Theta and $\mathrm{C}$ at $\mathrm{t}$, we calculate these 
values for $t+\Delta t$ as follows, after substitution of $i=1,2$, 3 ... L-1, where $\mathrm{L}=41$ corresponds to $\infty$.

Now equations (16) to (19) systems of equations is solved by Thomas Algorithm as discussed in Carnahan, Luthor and Wilkes [12]. Then $\theta$ and $\mathrm{C}$ are known for all values of $y$ at $t+\Delta t$. Replacing values of $\theta$ and $C$ in equations (18), (19) and solved by same with initial and boundary conditions (20), we have solutions for $\mathrm{u}$ and $\mathrm{v}$ form equations (16) and (17) till desired time t. CrankNicolson implicit finite difference method is second order method $\left(\mathrm{o}\left(\Delta \mathrm{t}^{2}+\Delta \mathrm{y}^{2}\right)\right)$ in time and has no limitation for space and time steps, that is, the method is unconditionally stable. Computation has been executed for $\Delta \mathrm{y}=0.1, \Delta \mathrm{t}=0.002$ and repeated till $y \max (=4)$ that corresponds to infinity.

\section{RESUlt AND DiscusSion}

With reference of physical problem, numerical results for dimensionless velocities $\mathrm{u}$ and $\mathrm{v}$, temperature $\theta$ and concentration $\mathrm{C}$ are discussed with help of graphs by assigning numerical values of thermal Grashof number Gr, solute Grashof number Gm, Schmidt number Sc, Prandtl number Pr, radiation parameter $\mathrm{R}$, magnetic parameter $\mathrm{M}$, permeability of porous medium $\mathrm{K}$, Chemical reaction parameter $\mathrm{Kr}$ and inclination angle $\lambda$.

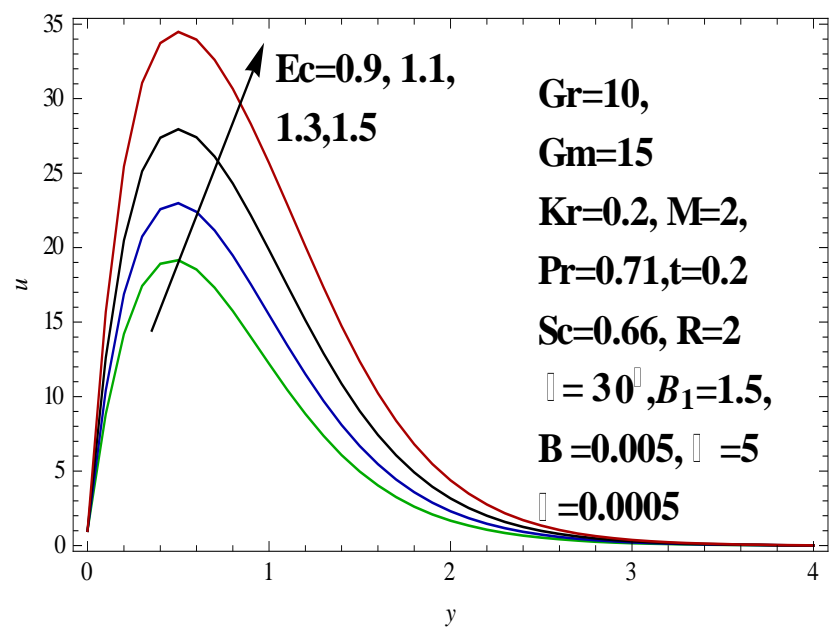

Figure 1. Velocity profile 'u' with values of "Eckert No."

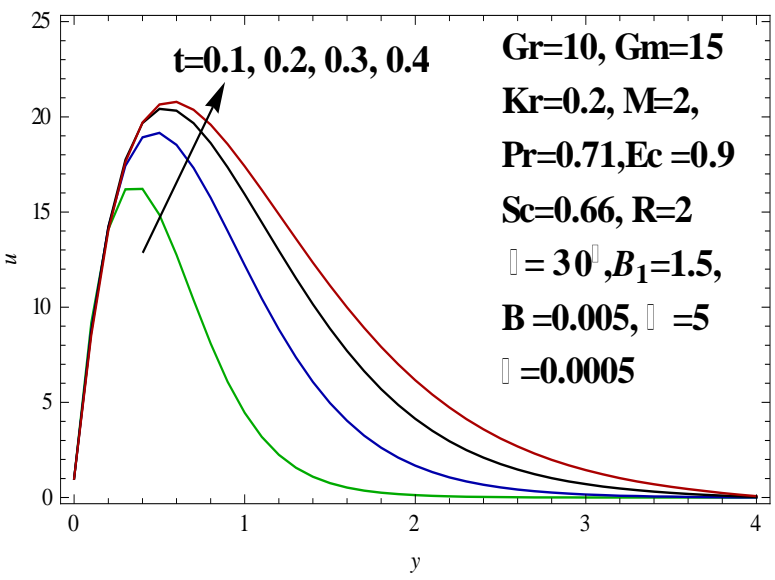

Figure 2. Velocity profile 'u' with values of "time" Figure 3.

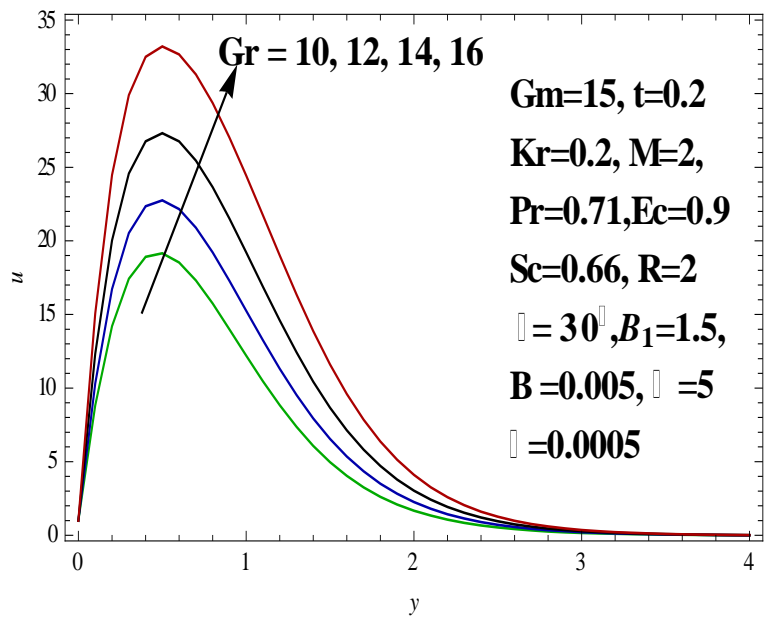

Figure 4. Velocity profile ' $u$ ' with values of " Grashoff No."

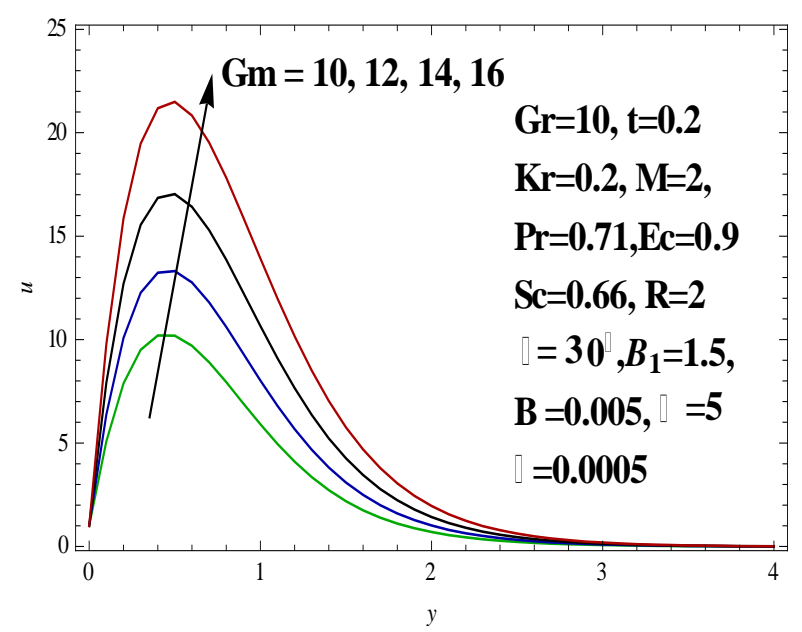

Figure 5. Velocity profile ' $u$ ' with values of " Solutal Grashoff No." 


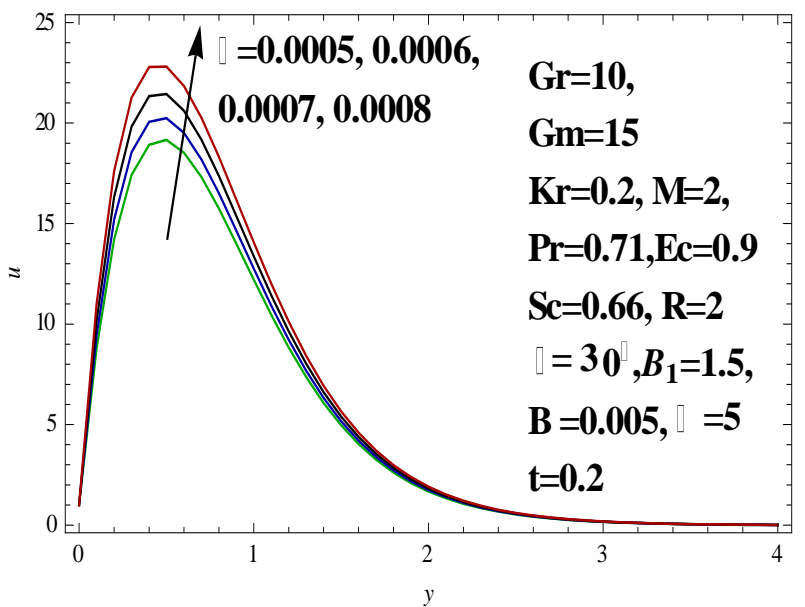

Figure 6. Velocity profile ' $u$ ' with values of "Viscoelastic parameter"

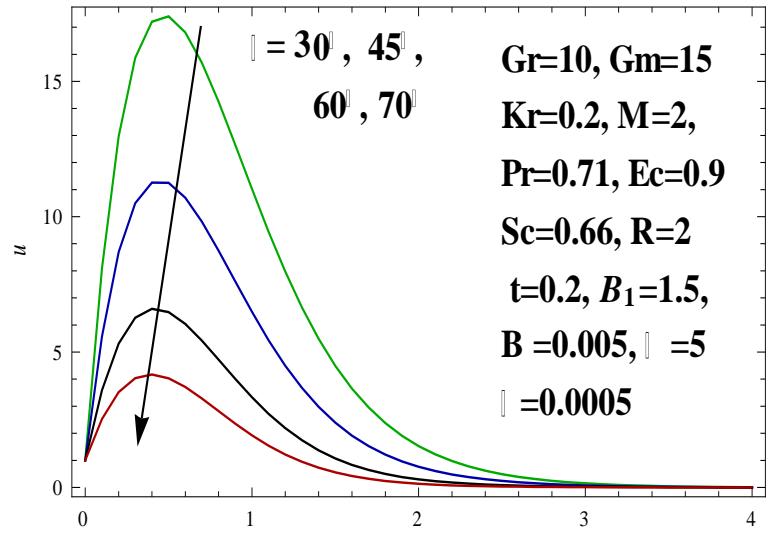

Figure 7. Velocity profile ' $\mathrm{u}$ ' with values of "Inclination angle"

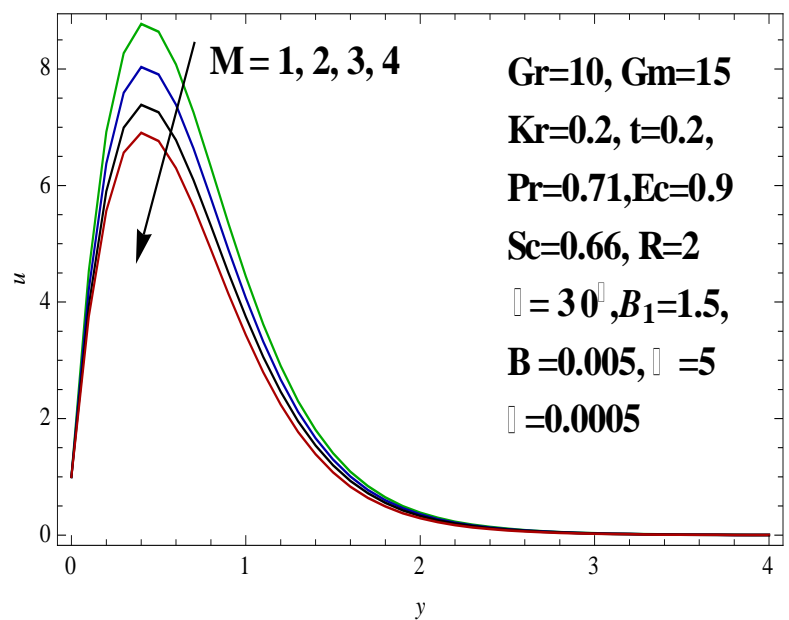

Figure 8. Velocity profile ' $u$ ' with values of "Magnetic parameter"

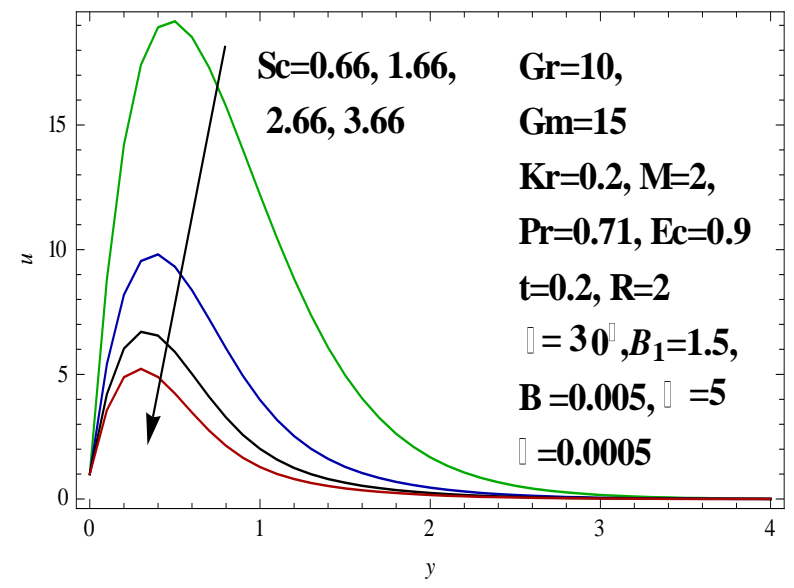

Figure 9. Velocity profile 'u' with values of "Schmidt Number"

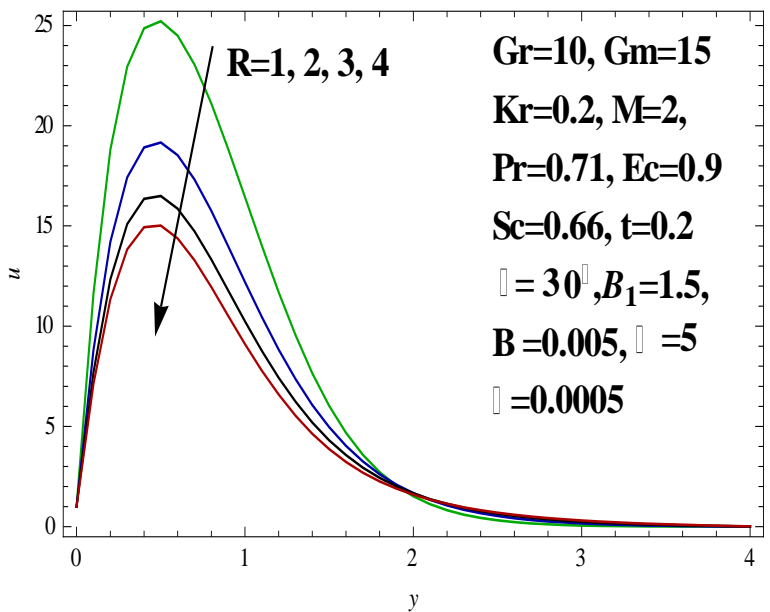

Figure 10. Velocity profile 'u' with values of "Radition parameter"

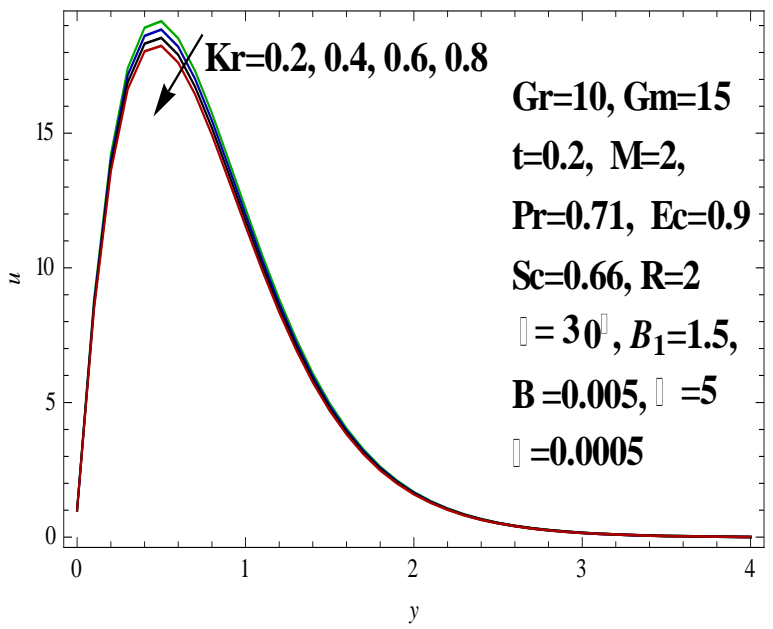

Figure 11. Velocity profile ' $u$ ' with values of “Chemical Reaction parameter" 


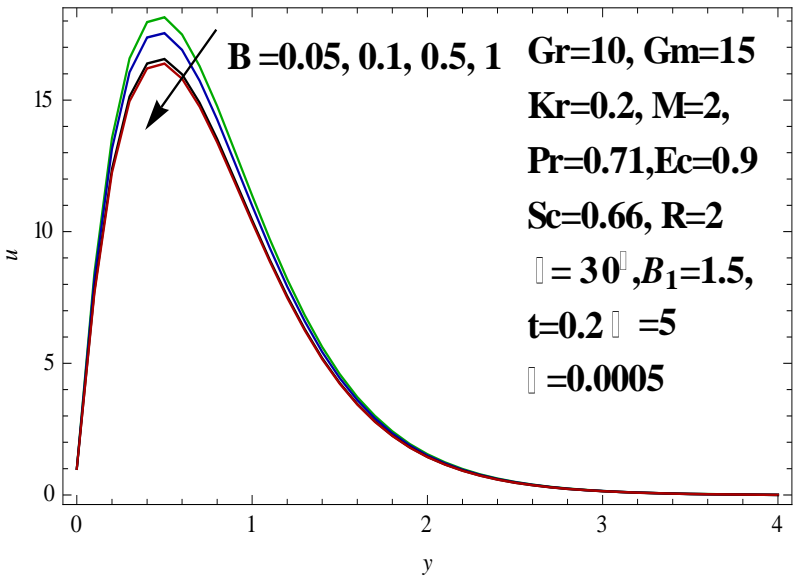

Figure 12. Velocity profile 'u' with values of "dust particle parameter"

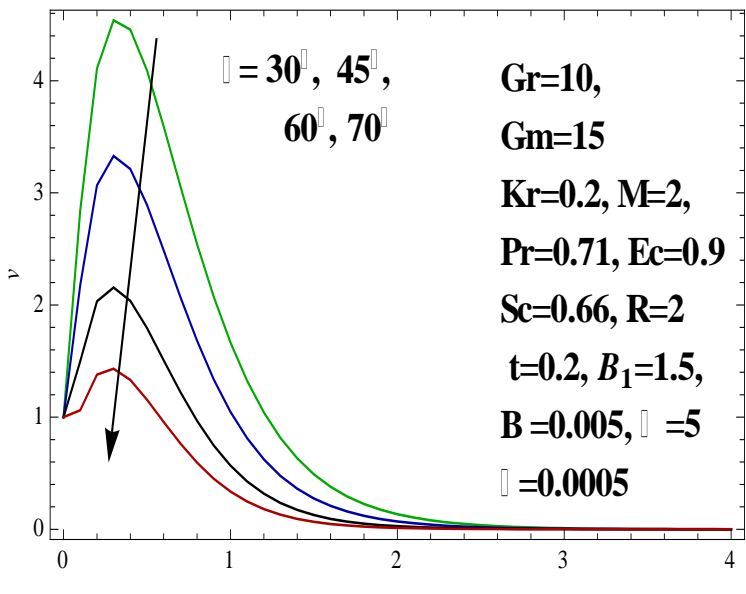

Figure 13. Velocity profile 'v' with values of "Inclination angle"

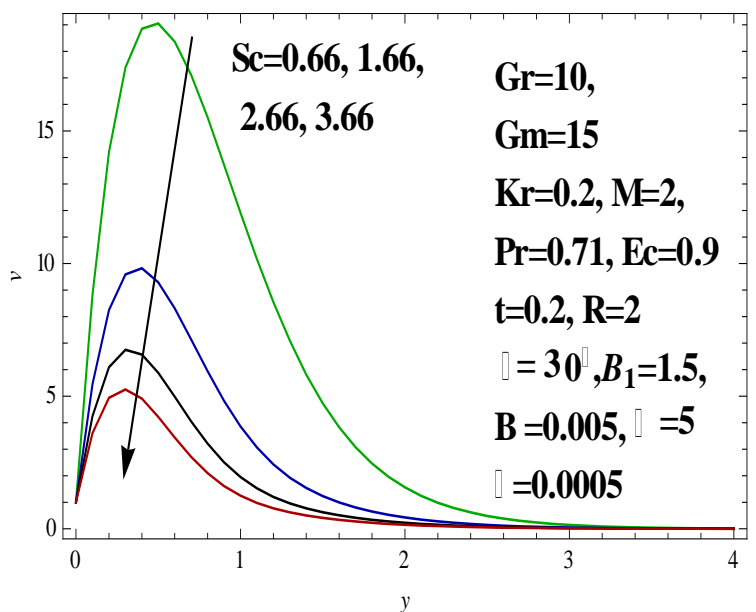

Figure 14. Velocity profile 'v' with values of " Schmidt Number"

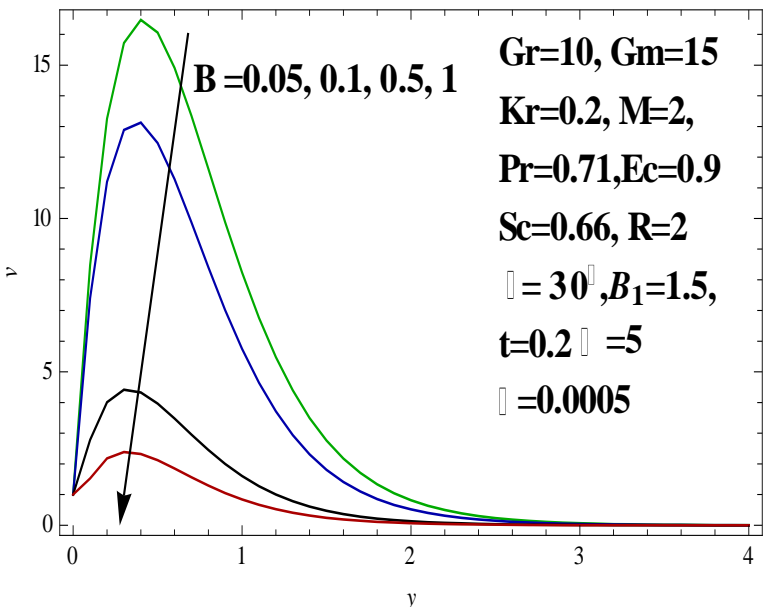

Figure 15. Velocity profile 'v' with values of “ dust particle parameter"

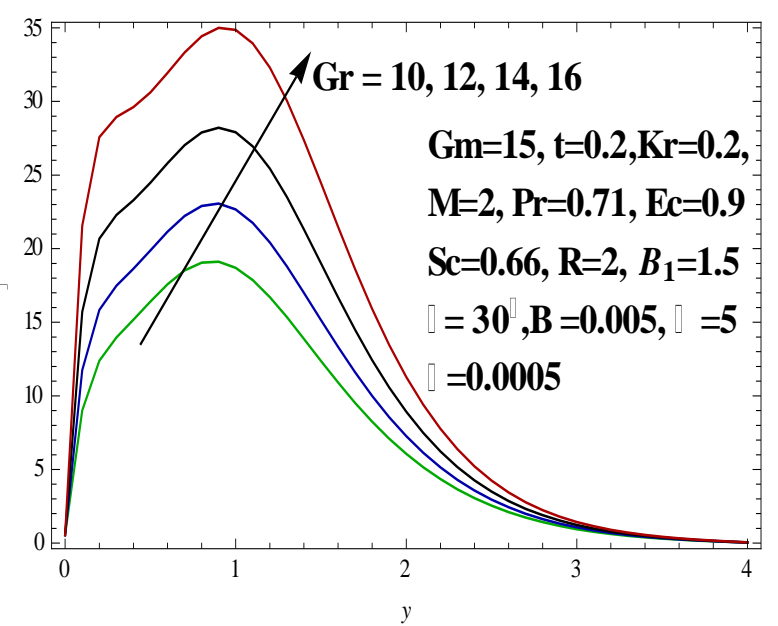

Figure 16. Temprature profile Theta with values of "Grashoff numberr"

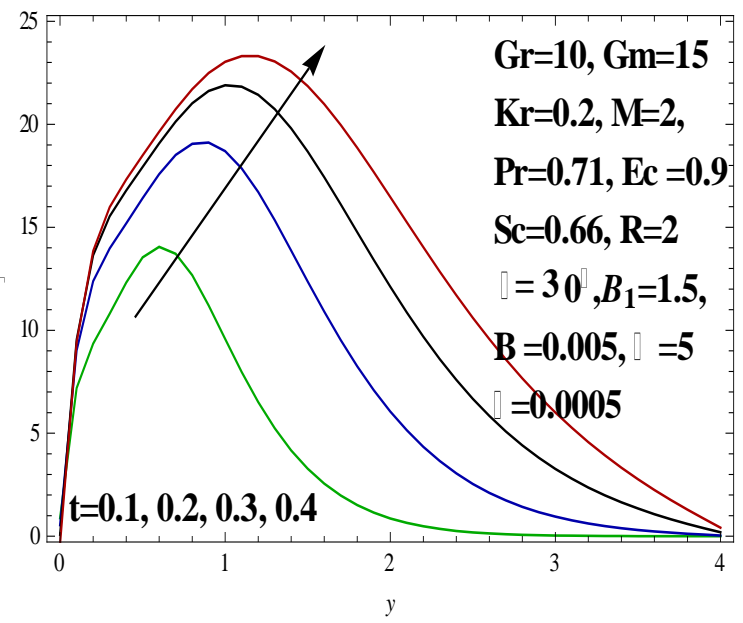

Figure 17. Temprature profile Theta with values of "time" 


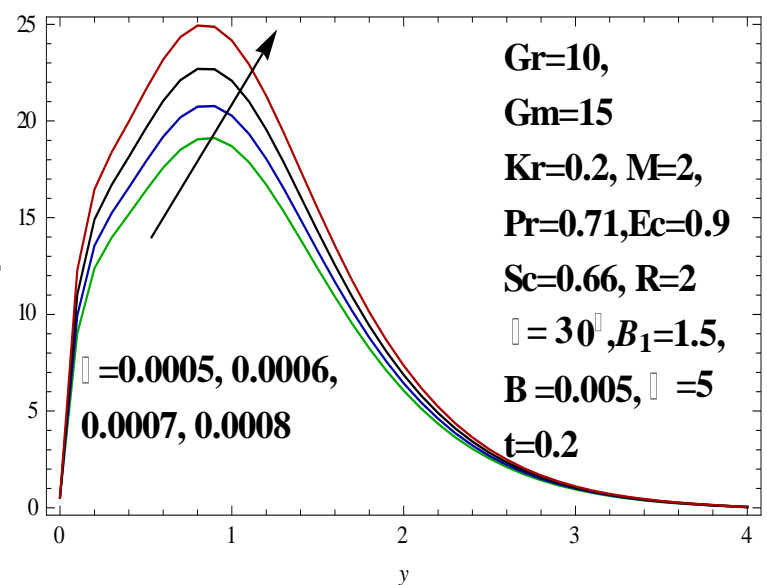

Figure 18. Temprature profile Theta with values of "Viscoelastic parameter"

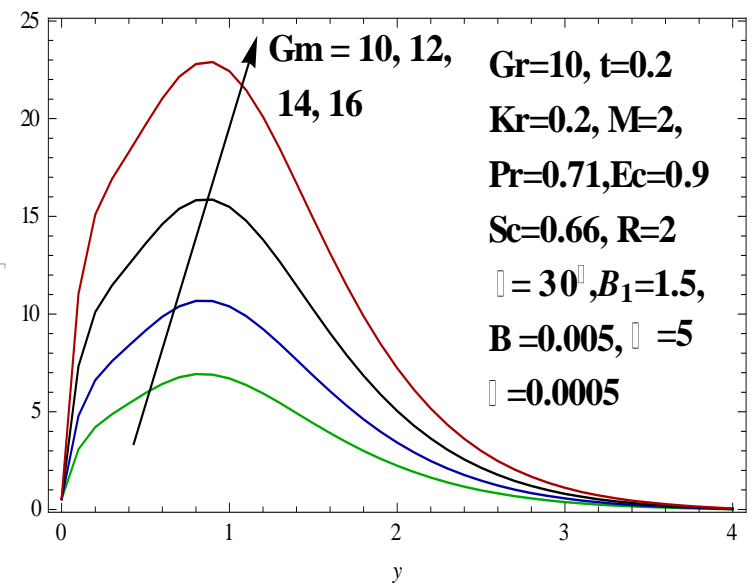

Figure 19. Temprature profile Theta with values of "Solutal Grashoff number"

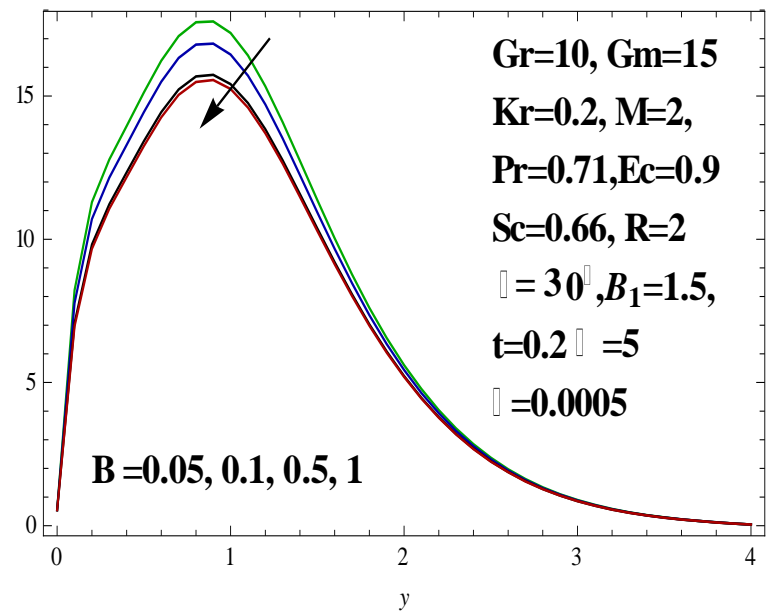

Figure 20. Temprature profile Theta with values of "Solutal Grashoff number"

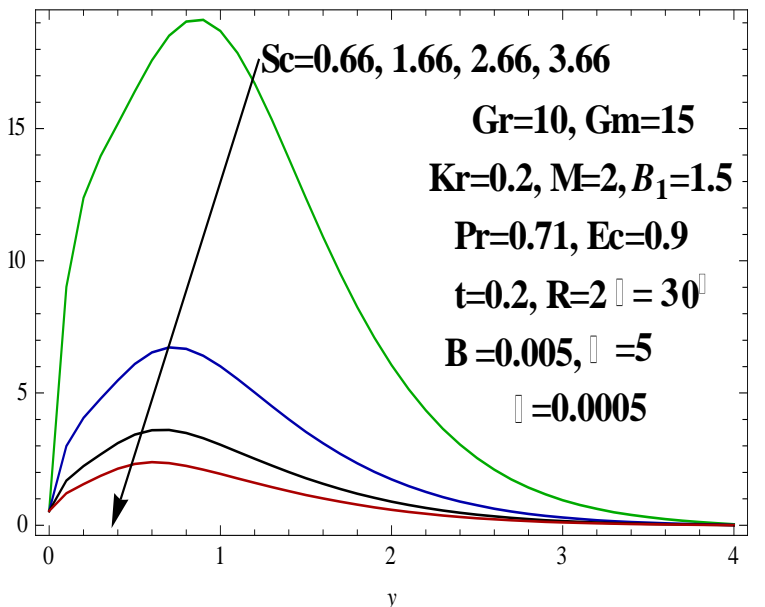

Figure 21. Temprature profile Theta with values of "Schmidt number"

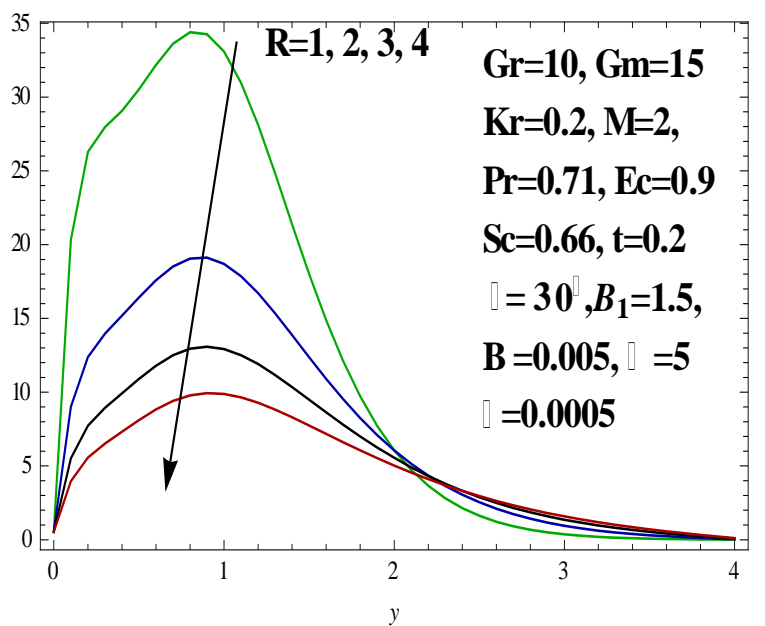

Figure 22. Temprature profile Theta with values of " Radiation parameter"

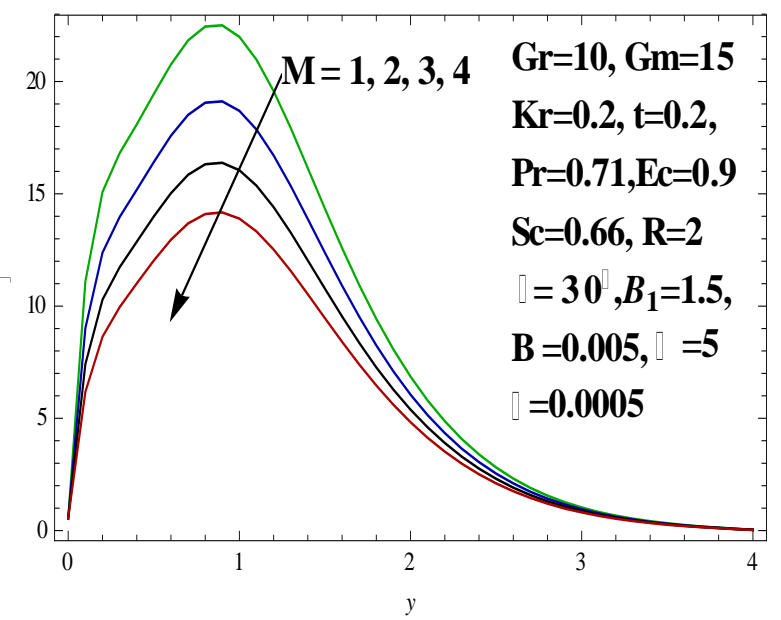

Figure 23. Temprature profile Theta with values of "Magnetic parameter" 


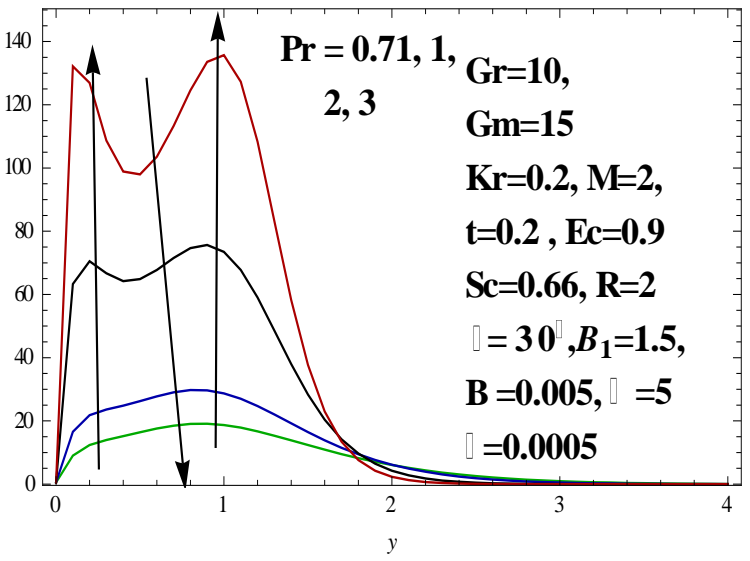

Figure 24. Temprature profile Theta with values of " Prandlt number"

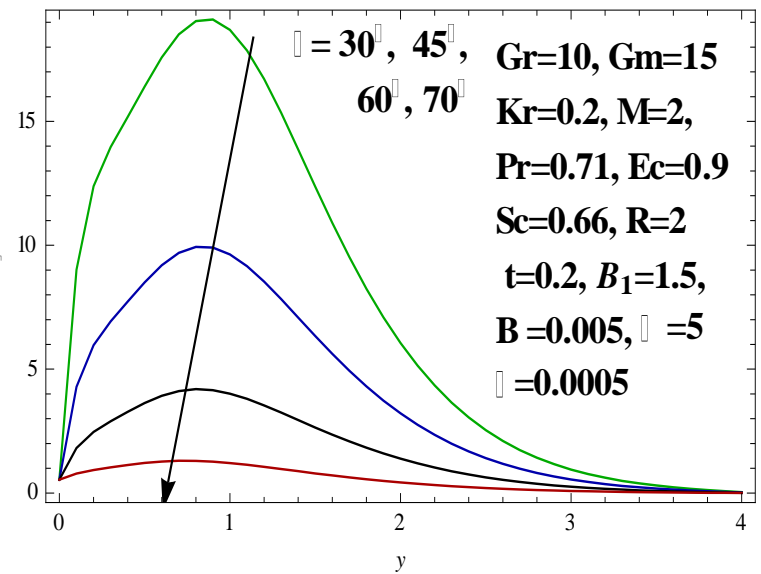

Figure 25. Temprature profile Theta with values of "Inclination angle"

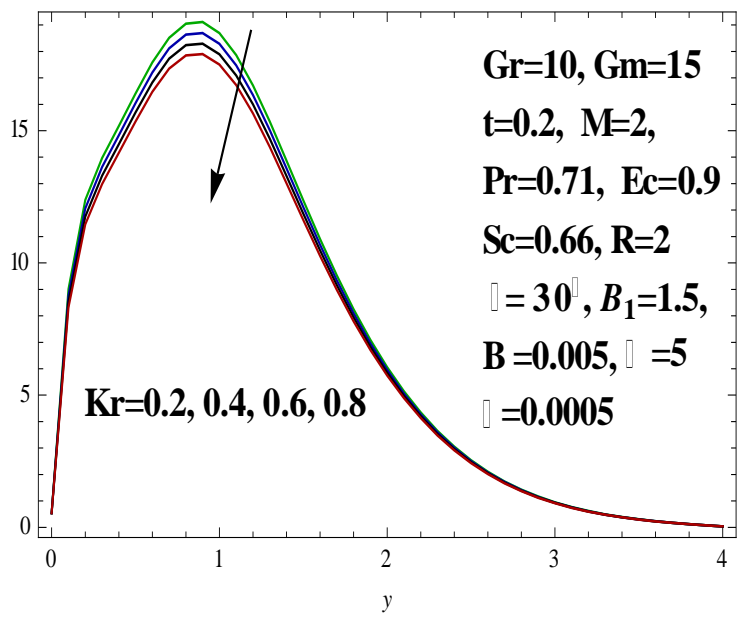

Figure 26. Temprature profile Theta with values of " Chemical Reaction parameter"

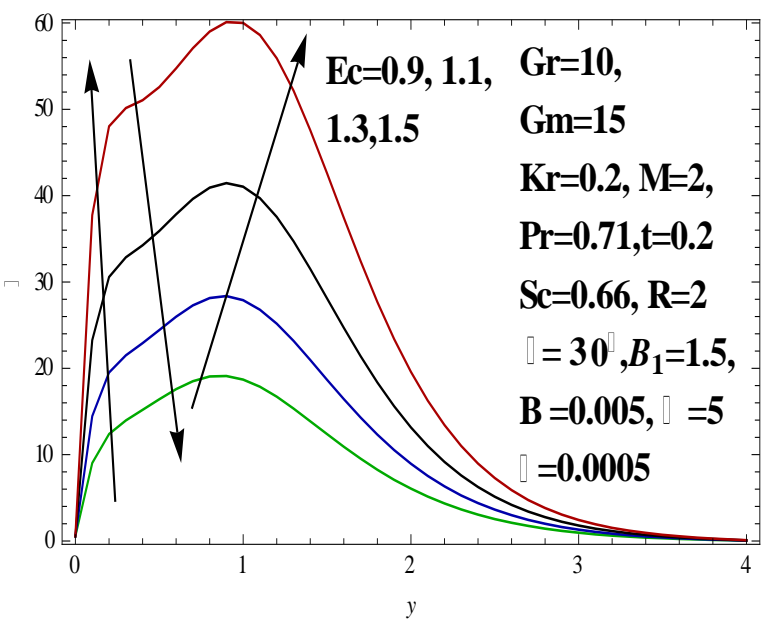

Figure 27. Temprature profile Theta with values of " Eckert number"

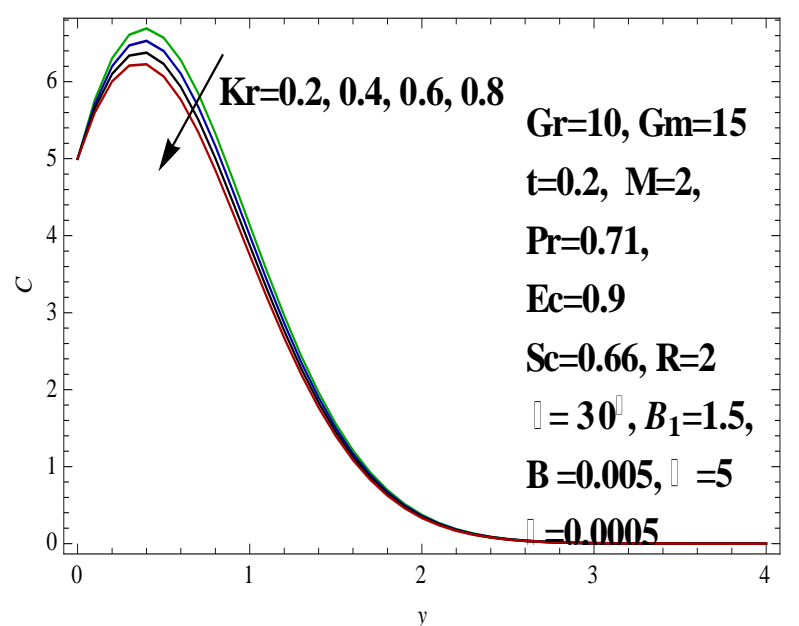

Figure 28. Concentration profile with values of " Chemical Reaction parameter"

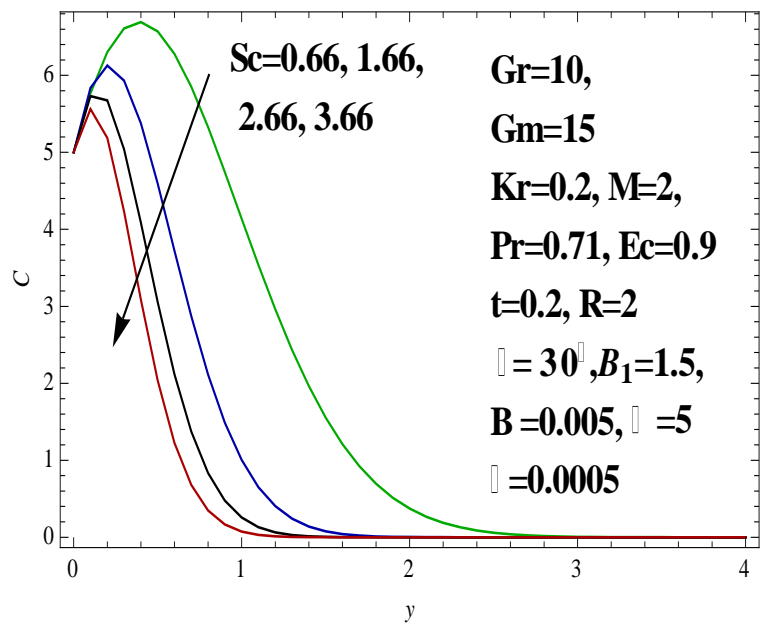

Figure 29. Concentration profile with values of "Schmidt number" 


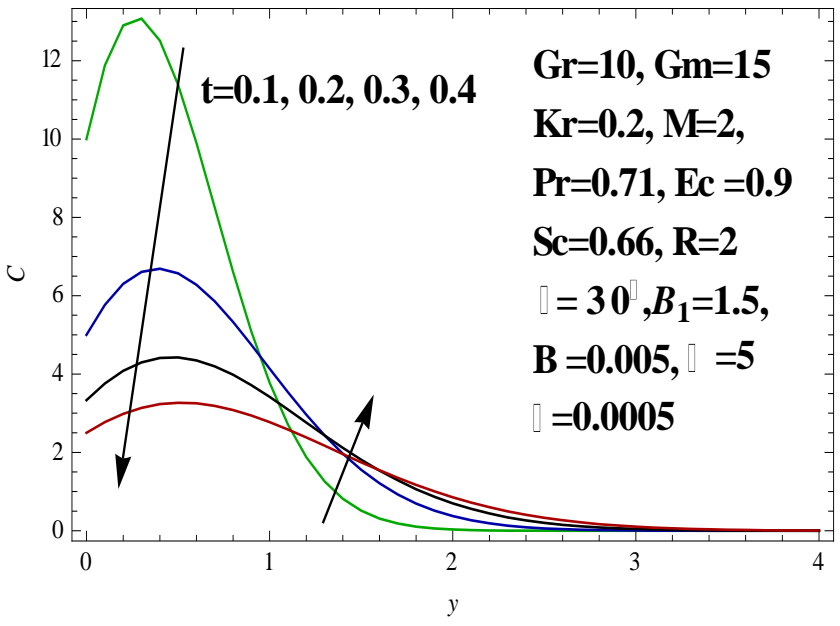

Figure 30. Concentration profile with values of "time"

In figure 1, 2, 3, 4 and 5 fluid velocity profile ' $u$ ' increases on increment in Ec, t, Gr, Gm and $\Gamma$ while decreases in figure $6,7,8,9,10$ and 11 on increment in $\lambda, \mathrm{M}, \mathrm{Sc}, \mathrm{R}, \mathrm{Kr}$ and $\mathrm{B}$.

The velocity of dust particles ' $\mathrm{v}$ ' behaves in like manner as of fluid particles. Some effects with the values of some parameters are shown. On increment in $\lambda$, Sc and $\mathrm{B}$, the velocity profile of dust particle decreases in figure 12, 13 and 14.

The Temperature profile Theta in figure 15, 16, 17 and 18 increases on increment in Gr, t, $\Gamma$ and $\mathrm{Gm}$. Temperature for short time increases and then decreases. Temperature increases sharply with increase in $\mathrm{Gr}, \mathrm{Gm}$ and $\Gamma$. And temperature in figure 19, 20, 21, 22, 23, 24, 25 and 26, decreases on increment of B, Sc, $\mathrm{R}, \mathrm{M}, \mathrm{Pr}, \lambda, \mathrm{Kr}$ and Ec. A special effect in figure 23 and 26 is seen, temperature increases sharply and decreases smoothly and then increases sharply.

In figure 27, 28 and 29 the concentration profile ' $\mathrm{C}$ ' decreases on increment in $\mathrm{Kr}, \mathrm{Sc}$ and t. Since the concentration is taken inversely to the time.

\section{CONCLUSIONS}

In this work we have concluded that the effects of radiation, chemical reaction, viscous dissipation and variable permeability for an unsteady MHD (Magnetohydrodynamic) Walter's-B viscoelastic dusty flow of an incompressible viscous and electrically conducting dusty fluid past a continuously moving inclined oscillating infinite porous plate embedded in porous media concluded the following conclusions:

a) Increasing inclination angle, velocities decreases rapidly.

b) Velocities increases with increase in Eckert number.

c) Velocity profile decreases with increase in radiation parameter.

d) The behaviors of dust particles are mostly same as of fluid particle. Velocity of dust particle decreases with increase in dust particle parameter.

e) Velocity profile increases with increases in viscoelastic parameter.

f) Temperature increases sharply and decreases smoothly and then increases sharply with Prandlt number and Eckert number.

g) Concentration decreases with increase in Schmidt number and Chemical reaction parameter.

h) Increment in Chemical reaction parameter results decrement in velocity of fluid.

i) On increasing magnetic parameter velocity of fluid decreases which validates the law of forces in fluid dynamics.

\section{REFERENCES}

[1] S. N. Bastia, R.S. Rath, "Steady flow and heat transfer in a viscoelastic fluid between two coaxial rotating disk", Proc. Mathematical Sciences. Vol.87, Issue9, pp.227-236, 1978.

[2] A. A. Raptis, H. S. Takhar, "Heat transfer fromflow of an elastico-viscous fluid", Int Comm. Heat and Mass Transfer, Vol.16, Issue.2, pp.193-197, 1989.

[3] R. C. Chaudhary, P. Jain, "Hall effect on MHD mixed convection flow of a viscoelastic fluid past an infinite vertical porous plate with mass transfer and radiation", Theoretical and Applied Mechanics, Vol.33, Issue.4, pp.281-309, 2006.

[4] K. Rajgopal, P. H. Veena, V. K. Pravin, "Non similar solutions for heat and mass transfer flow in an electrically conducting viscoelastic fluid over a stretching sheet saturated in a porous medium with suction/blowing", J. Porous Media, Vol.11, Issue.2, pp.219-230, 2008.

[5] S. Dholey, A. S. Gupta, T. R. Mahapatra, "Momentum and heat transfer in the magnetohydrodynamic stagnation point flow of a viscoelastic fluid towards a stretching surface", Mechanica. Vol.42, Issue.3, pp.263-272, 2007.

[6] P. Ganesan, V. M. Soundalgekar, "Finite difference analysis of transient free convection with mass transfer on an isothermal vertical flat plate", Int. J. of Engng. Sciences. Vol.19, Issue.6, pp.757-770, 1981.

[7] N. Pandya, A. K. Shukla, "Soret-Dufour and radiation effects on unsteady MHD flow past an impulsively started inclined porous plate with variable temperature and mass diffusion, Int. J. of Math. and Sci. Comp. Vol.3, Issue.2, pp.41-48, 2013. 
[8] N. Pandya, A. K. Shukla, "Soret-Dufour and radiation effects on unsteady MHD flow over an inclined porous plate embedded in porous medium with viscous dissipation", Int. J. Adv. Appl. Math. And Mech. Vol.2, Issue1, pp.107-119, 2014.

[9] U. R. Rao, V. R. Prasad, G. Vishwanath, B. Vasu "Radiation effects on unsteady free convection heat and mass transfer in a Walter's-B viscoelastic flow past an impulsively started vertical plate", Int. J. of Scientific \& Engineering Research, Vol. 3, Issue.8, pp.1-9, 2012.

[10] N. Pandya and R. K. Yadav "Numerical Simulation of SoretDufour and Radiation effects on Unsteady MHD flow of Viscoelastic Dusty fluid over Inclined Porous Plate", International Journal of Computer Sciences and Engineering, Vol. 6, Issue 6, pp. 898-908, 2018

[11] T. G. Cowling, "Magnetohydrodynamic", Intersicence Publisher, New York, 1957.

[12] Brice Carnahan, H. A. Luthor, J. O. Wilkes, "Applied Numerical Methods", John Wiley and Sons, New York, 1969.

\section{AUTHORS PROFILE}

Dr. Nidhi Pandya is my supervisor and Assistant Professor in the department of Mathematics and Astronomy, University of Lucknow, Lucknow, Uttar Pradesh, India. Now a day she is working on MHD viscous and viscoelastic flow in porous medium.

Mohammad Suleman Quraishi is a Research Scholar under the supervision of Dr. Nidhi Pandya. He is pursuing $\mathrm{Ph}$. D. degree from University of Lucknow.
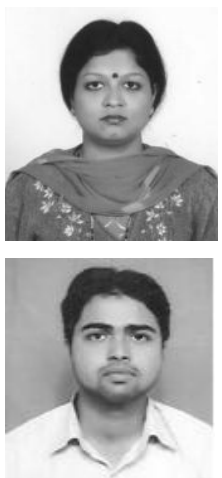